\title{
39 \\ FROM INTRA-ENTERPRISE TOWARDS EXTRA-ENTERPRISE PRODUCTION PLANNING
}

\author{
Aleš Ř́ha, Michal Pěchouček, Jiří Vokřínek, Vladimír Mařík \\ Gerstner Laboratory, Department of Cybernetics \\ Czech Technical University in Prague \\ Technická 2, Prague 6, 16627 Czech Republic \\ \{riha, vokrinek, pechouc, marik\}@labe.felk.cvut.cz
}

\begin{abstract}
In the domain of production planning multi-agent systems there is a strong demand for extending the solution beyond the borders of a single enterprise in order to meet future business requirements: (i) to manage the extra-enterprise production planning effectively, (ii) to create and reconfigure flexible dynamic configuration of virtual enterprises (e.g. among collaborating business partners). The concept of extra-enterprise agents helps to facilitate secure remote access and manage the production planning processes from outside the factory, inspect the current and the future load of particular production units, modify already running customer-projects, or include/invoke a new customer-project into the system remotely.
\end{abstract}

\section{INTRODUCTION}

The application domain of the research described here is oriented to support a project-oriented type of production. Unlike mass-production, in the case of the project-oriented production there is always a limited series of complex products of one type manufactured (e.g. space shuttles, power turbines, TV broadcasters or patterns and forms). Accordingly, an important part of resources has to be devoted to design-related activities such as quotation and configuration, design, and production planning. Production-oriented planning consists of three separate (while interrelated) phases:

- quotation and configuration, where the quotation engineer negotiates with a customer on a detailed specification of the product [1],

- project specification, where the complete product configuration is transformed into specifications of a project and required resource and

- resource allocation, where the required resources are allocated in time to appropriate resource providers.

Even though different areas of artificial intelligence, such as constraint programming, theorem proving, or evolutionary computing [2] very often support the first phase, we have relied on the human expertise in both the quotation and configuration as well as the project specification phases. The research described in this paper concerns primarily the last phase, where appropriate resource providers, such as machines, technicians, departments etc., allocate the required amount of 
resources. It becomes rather difficult to allocate project-related resources provided by partially booked resource providers and to allow re-planning due to resource malfunction or due to scheduling of higher priority projects. In this paper we will comment how the ExPlanTech [3] multi-agent technology addresses both the intraenterprise and extra-enterprise aspects of the resource allocation problem.

\section{INTRA-ENTERPRISE PRODUCTION PLANNING}

ExPlanTech [3] is a physically distributed multi-agent system that exploits latest research achievements in the fields of distributed artificial intelligence, agent based computing and production planning. It builds upon the theoretical concept of social intelligence representation, acquaintance models, meta-agents and community monitoring, agentification and others. These concepts were originally studied, implemented and tested in the ProPlanT multi-agent system [4]. A full, stable and approved implementation of the ExPlanTech system has been partially supported within the ExPlanTech project (IST-1999-20171). The resulted multi-agent system has been implemented in JAVA and complies with the latest standards in agent based computing - FIPA (Federation of Intelligent Physical Agents - http://www.fipa.org/) [6]. The JADE (Java Agents Development Environment sharon.cselt.it/projects/jade/) platform has been adopted [7]. The system has been tested in the Liaz Pattern Shop factory that produce moulds, forms and dies.

ExPlanTech was originally designed for the purposes of intra-enterprise production planning in the project oriented manufacturing environment - especially for managing resources and estimating delivery times and costs of a possible project. ExPlanTech integrates existing software systems that administer the production processes within the enterprise (such as enterprise resource planning systems (ERP), material management, human resource systems, CAD/CAM systems) by the agentification process. The agentified software system is encapsulated within the agent wrapper that administers agent-to-agent communication and collects the agents' social knowledge [5]. Such a software system becomes an agent - a fullyfledged member of the multi-agent system. The factory management does not need to replace the entire operational information systems by a new technology. Instead, they can make a best use of a combination of (i) the software infrastructure they already have and (ii) the novel, agent-based production planning technology.

The organizational structure of the ProPlanT (and also ExPlanTech) multi-agent system mirrors the organizational structure of the given enterprise. The agents have been divided into two fundamental super-classes: intra-enterprise (IEA) agents and extra-enterprise (EEA) agents. We distinguish among the following basic classes of IEA agents: Production Planning Agent (PPA), which is in charge of the product configuration and project specification, Production Management Agent (PMA), which accounts for decomposition of the project/task into several (sub)tasks and for contracting the best possible collaborator and Production Agent (PA), which belongs to the lowest level production units that simulate or encapsulate shop floor production processes. 
Meta agent (MA) - a special monitoring agent visualises information, material and work flows across the agents' community. Shall be noted that the operation of the community of agents is completely independent of the meta-agent. Besides monitoring, the meta-agent can carry out independent reasoning and analyse the community overall behaviour. Such reasoning may result in suggestions aimed at efficiency improvements.

Apart from agentification and meta-agents, there is another important theoretical concept that is a vital part of the ProPlanT/ExPlanTech technology. The agents' wrappers are equipped with an acquaintance model that is a social knowledge container. A social knowledge of an agent (its computational model of behaviour of the rest of the multi-agent system) allows accurate while fast production planning, efficient re-planning in the case of a malfunction, or on-line community reconfiguration when some of the encapsulated software/hardware components fail to operate [5].

While integrated within the company internal ERP system, ExPlanTech elaborates an optimal production plan for a particular project. The plan, which is visualized in the graphical form, is provided to a human decision maker who can either confirm the plan or invoke an automated re-planning. The production plan can be re-planned either by changing priorities of the particular project, increasing or decreasing capacities of the respective production unites or redefining project specification. After testing in the factory floor, it has been identified that this technology provided more optimal production plans than the human planning expert. This is why the management can be provided with more accurate estimations of the required resources and due dates, by which the overall competitiveness of the factory increases and resources are used in the most optimal way.

\section{EXTENDED ENTERPRISE}

The optimal production plan should balance the available resources, maximizing the number of orders to be processed and minimizing their due times. As the workshops capacity is not unlimited, the decision has to be made whether the specific task/subtask will be implemented internally or subcontracted externally (e.g. within the frame of a virtual enterprise). Such a decision might be crucial in order not to threaten successful completion of other orders and not to misbalance the whole production flow. The efficient supply chain management can be handled by extending the original agent-based solution out of the boundaries of a single enterprise [8]. Moreover, orders are categorized by an internal priority value ensuring that orders with a higher priority are manufactured before those with lower ones. The ExPlanTech system monitors loads of workshops and other resource providers and consequently it 'measures' how suitable is the requested order. 


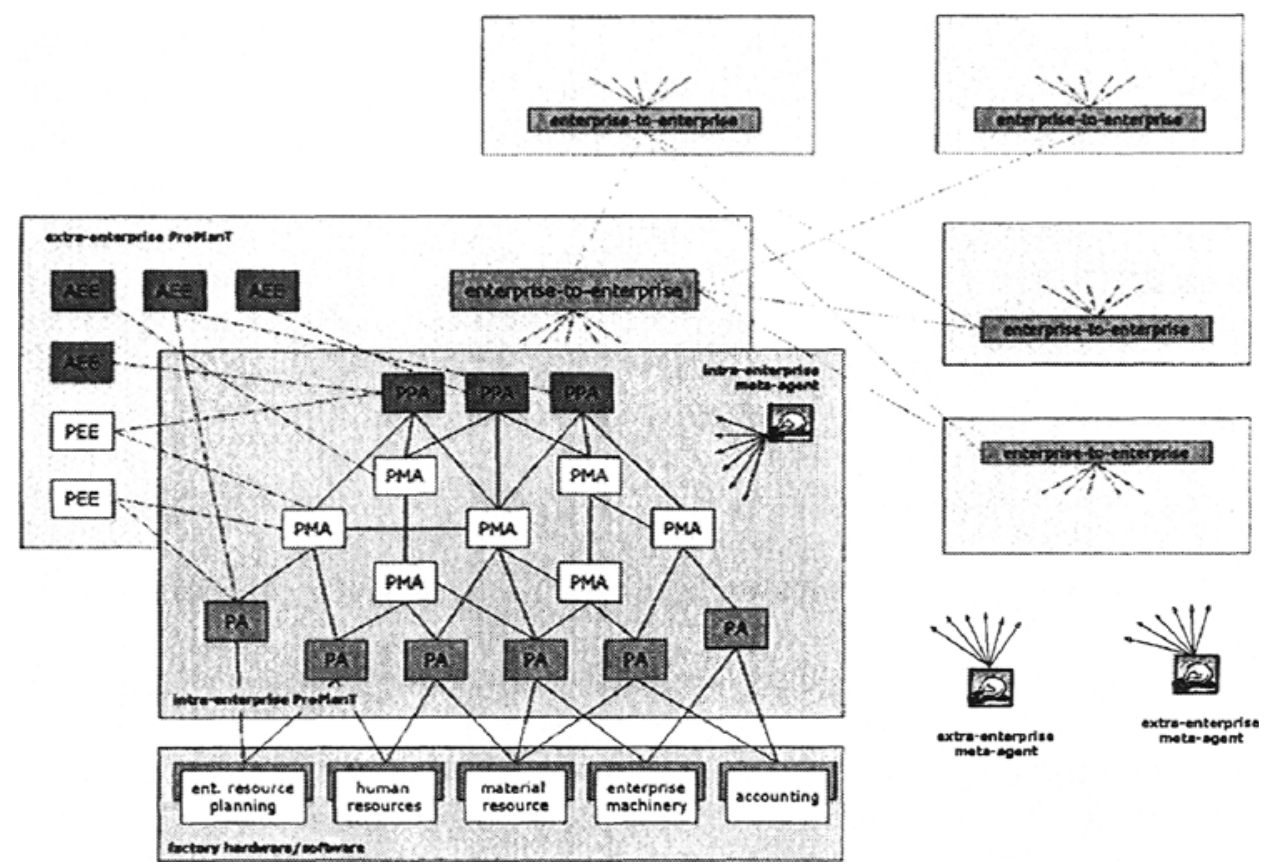

Figure 1: ProPlanT/ExPlanTech Architecture for Intra/Extra-Enterprise Production Planning

The extra-enterprise concept of the ExPlanTech technology provides several other classes to the agent community (see Figure 1):

- Passive Extra-Enterprise Agent (PEE) which may passively access the production planning data from outside of the company. These data can be accessed either by (i) customers, who (if the access rules permit) may inspect the status of their order manufacturing or (ii) managers who may want to monitor the load of production unites and available resources,

- Active Extra-Enterprise Agent (AEE) which may trigger from outside of the enterprise the entire course of production planning. AEE agent negotiates with the PPA agent in order to specify the production requirements, the deadline and the budgetary constraints. It can also subcontract individual PMA or PA agents for only parts of the products.

- Enterprise-to-Enterprise Agent (E2E) connects two enterprises that want to participate in a supply chain. E2E agent models the cooperating enterprise as an instance of a PA agent. The E2E agent acts on behalf of the cooperating enterprise and allows the PMA or PPA agents to subcontract it for a specific part of the supply. The E2E agentifies the whole enterprise.

Either a user or the AEE agent usually communicates with the PPA agent. The PPA agent constructs a component list and delegates further responsibilities to the PMA agents who contract either the best possible PA agents or via the E2E agent the best possible supplier or a collaborating entity. Costs and due times, that represent the distributed production plan, are then back-propagated to either the AEE agent or the user. 
The extra-enterprise agents can be appreciated either by production managers when traveling (monitoring of the production, competitive planning etc.) or by customers (observing production progress of their orders). The extra-enterprise (EE) technology provides accessibility of the production planning data to the management of the enterprise while they are out of the enterprise using modern information/communication standards. This technology is primarily designed for accessing the production data (occupancy of workshops, list of orders, material etc.) via the Internet by an ordinary web browser. Apart from the portable or the desktop PC, the extra-enterprise agents can be installed on the mobile PDA devices that are based on the Windows CE platform. The extra-enterprise agents' wrappers can be adapted for communication using the Wireless Application Protocol (WAP) for running in the cell-phone devices. Only authenticated agents/human users can access and manage the intra-enterprise production planning data.

The E2E agent extends the ExPlanTech technology for managing outsourcing and capacity sharing across various enterprises. In the simple case, a supplier/collaborator can be represented as a virtual workshop by a simple E2E agent that advertises (and administers) only the services and capacities it is able to provide. Such an E2E agent represents either one physical workshop or can be viewed as a wrapper of the entire collaborating enterprise. The shared (public) knowledge about currently available resources should be prepared in a standard form with respect to the agreed and shared knowledge ontology. The agent could, for example, read the data from the partner's enterprise database or could be provided with this data by the ERP system. If there are not enough resources locally available, the planning system is able to contract such an agent and avoid failing to meet the deadline.

The second option is to use the E2E concept for interconnecting several enterprises that use the ExPlanTech technology on the intra-enterprise level. In such a case, the E2E agent does not simply represent services, the collaborator offers to the community, but serves as proxy to its own multi-agent community. Each intraenterprise ExPlanTech system maintains one E2E agent that periodically checks with its intra-enterprise agents for their available resources and advertises these to the other E2E agents. Once the production planning agents within one enterprise are threatened by a possibility of failing to meet the deadline, they immediately contact their E2E agent which initiates negotiations with the other E2E agents representing the outsourcing resources and finds the most suitable subcontractor.

\section{EXTRA-ENTERPRISE PLANNING SCENARIOS}

Firstly, we will comment the technology migration phase when the agent-based system is integrated within the factory information infrastructure. Initially, the set of orders is defined in the factory information system. Orders specifications are propagated to the PPA agent, that starts planning by broadcasting the requests to the set of PMAs according to the specifications of the orders. Each order is represented in the planning system by a partially ordered set of tasks, required resources and priority of the order. The PMAs start negotiating aimed at finding the best configuration of the PAs for each task. The PA schedules the tasks on their resources. The final plan is propagated through the community back to the information system. As the result of this initial stage, the system contains all the 
orders to be carried out in the factory and is ready for receiving (planning) new orders.

\subsection{Passive Access}

The current status of the production planning system can be inspected via an ordinary internet browser, mobile PDA, or WAP cell phone using the passive extraenterprise agents (see Figure 2). These agents are created and connected to the multiagent system in real time. According the access rights, the customers can inspect "their own" orders or the company managers can access all the orders being processed by the agents. This information is downloaded directly from the agents via standard inter-agent communication. It is possible to inspect plans for the selected tasks or the entire load allocation of some PA (workshop, machine). For example the plan for the task "Z_211-0005 Seat model" can be seen only by the customer from the company "AUTO GD" or by the factory manager.

\subsection{Active Access}

Active extra-enterprise agents can be created and connected to the system in the same way as the passive extra-enterprise agents. The AEEs provide convenient functionality to factory managers. The manager, when traveling, can define a new order on the server side and ask the system to plan it. According to the resulting plan, the system either books the capacities - permanent planning (by sending the achieve speech-act messages) or, more often, only checks possibility of acceptance of a new order (by sending the query speech-act messages) - competitive planning. The competitive planning can be used for comparing alternative plans for several tasks. In the case of the 'Seat model' task, the manager (or a trusted customer from the "AUTO GD" company) can try to plan production within the manufacturing enterprise or with subcontracting parts of the project externally (e.g. using cooperating CAD studio). The manager will find out differences between two obtained plans and decides not to use external resources for the "Z_211-0005 Seat model" and will plan this order permanently on internal resources only (See Figure 2).

\subsection{E2E Connecting}

The enterprise-to-enterprise agent is used for linking suppliers into a virtual enterprise. The cooperating enterprise can offer the available resources via the E2E agent. Other enterprises can use these resources transparently like their own resources. An E2E agent plays the role of trustful active EEA on the side of one enterprise and the role of the PA on the side of the other enterprise. The planning system can only operate with available resources of the other enterprise that are accessible via the E2E. For example, the cooperating enterprise (CAD studio) integrates their resources within the factory via $\mathrm{E} 2 \mathrm{E}$ representing $\mathrm{CAD}$ studio in the virtual enterprise and can be used instead of the standard factory department ("CAD konstrukce") when it is more efficient. 


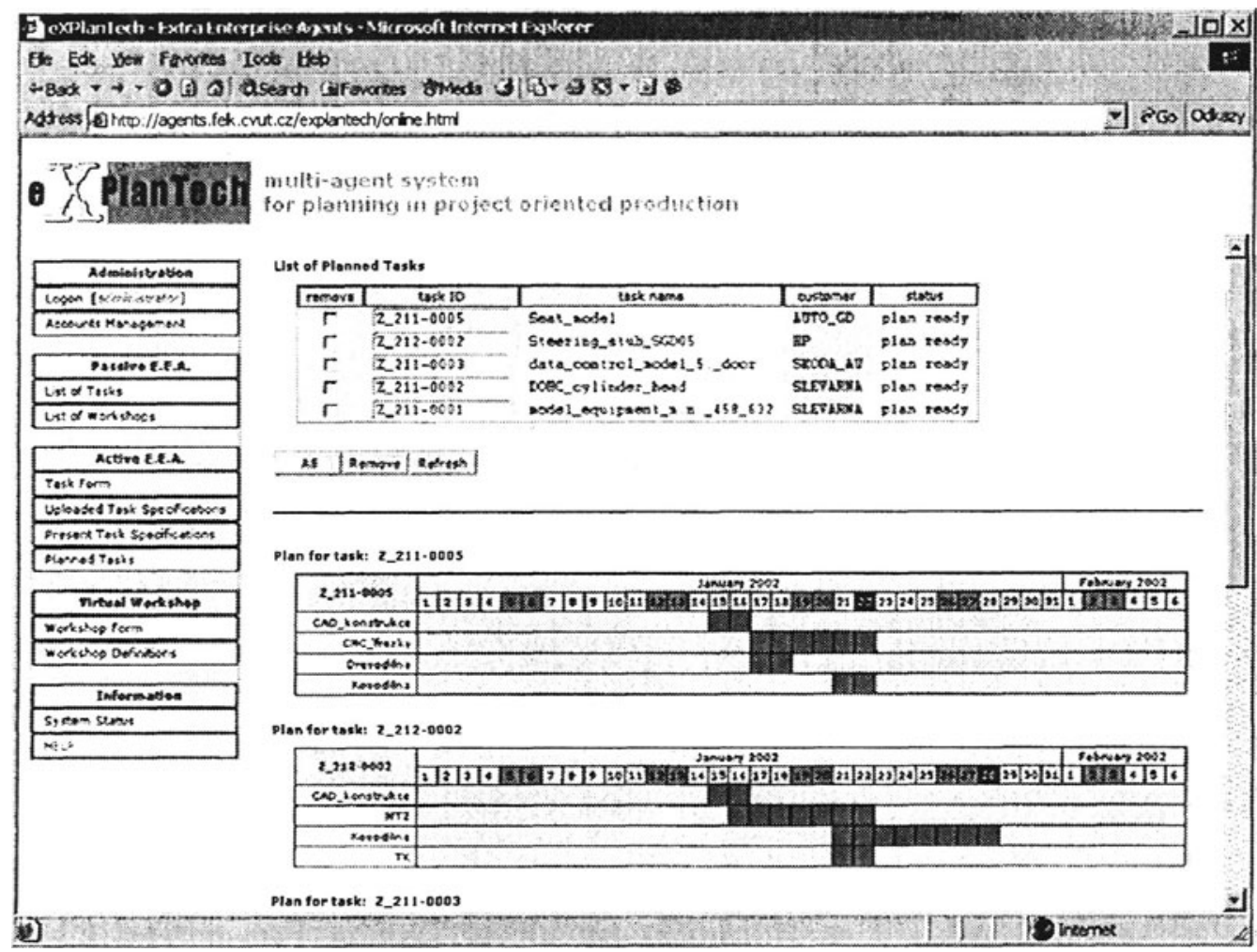

Figure 2: Screenshot of the internet browser window. On the left there is an access panel to the functionality of the PEE, AEE and E2E agents, while in the main frame, there is a result of the selected action - a list of planned tasks from AEE agent.

\section{CONCLUSIONS}

In this paper we have illustrated strengths of the multi-agent approach to intraenterprise project-driven production planning and potentials of extending the general technology to the extra-enterprise level. The ExPlanTech multi-agent community has been originally designed so that each agent represents/models a particular manufacturing unit within the respective factory (e.g. department, workshop, CNC machine, warehouse). Agents' interaction simulates possible variations of the course of manufacturing. Such a model facilitates interaction-based production planning and flexible, intelligent replanning/reconfiguration once a production unit fails to operate, gets overloaded or simply a high priority order is in conflict with an already planned order. The agents may represent not only the in-house production actors but also other members of the supply chain which contribute to manufacturing of a required product. This is why the concept of agentification that has been designed for integrating legacy systems and encapsulating hardware machinery can be used for integrating suppliers as well. 
This research work has been supported by the European Commission contract No. IST-1999-20171 (ExPlanTech) and by the Ministry of Education, Youth and Sports of the Czech Republic within the frame of the project No. MSM212300013.

\section{REFERENCES}

[1] Wielinga, B. J. and Schreiber A.:Configuration-design Problem Solving. IEEE Expert, 12(2), 1997, pp. $49-56$

[2] Kubalík, J., and Lažanský, J.: Genetic Algorithms and their Tuning. In: Proceedings of CASYS '98 (Dubois D., ed.), Liege, Belgium, 1998, pp. 217-229

[3] Ríha, A., Pěchouček, M., Vokřínek, J. and Mařík, V.: ExPlanTech: Exploitation of Agent-based Technology in Production Planning. In: V. Mařík, et. Al, (Eds.), Multi-Agent Systems and Application II, LNAI 2322, Springer Verlag, 2002, pp. 308-322

[4] Mařík, V., Pěchouček, M., Štěpánková, O., and Lažanský, J.: ProPlanT: Multi-Agent System for Production Planning. Applied Artificial Intelligence, vol. 14, No.7(2000), pp.727-762

[5] Pěchouček, M., Mařík, V., and Štěpánková, O.: Towards Reducing Communication Traffic in Multi-Agent Systems. In: Journal of Applied System Studies, Cambridge International Science Publishing, Cambridge, UK, No.1, 2001

[6] FIPA: Agent Management. In: http://www.fipa.org, Geneva, Switzerland, 1998

[7] Bellifemine, F., Poggi, A., and Rimassa, G.,: Developing Multi-agent Systems with JADE. In: Seventh International Workshop on Agent Theories, Architectures, and Languages (ATAL-2000), Boston, MA, 2000

[8] Camarinha-Matos, L.M., Afsarmanesh, H., and Lima, C.: Hierarchical Coordination in Virtual Enterprises. Journal of Intelligent and Robotic Systems, vol. 26 (1999), Issue 3/4, pp. 267-287 